\title{
Boundary-limited Thermal Conduction of Crystalline Rods Oriented near Phonon-Focusing Caustics
}

\author{
A. G. Every ${ }^{1}$ and A. A. Maznev ${ }^{2}$ \\ ${ }^{1}$ School of Physics, University of the Witwatersrand, PO Wits 2050, Johannesburg, South Africa \\ ${ }^{2}$ Department of Chemistry, Massachusetts Institute of Technology, Cambridge, Massachusetts \\ 02139, USA
}

\begin{abstract}
We apply the Casimir model for boundary-limited heat conduction to single-crystal rods oriented near phonon-focusing caustics. We show that rods with axes close to the direction of an external conical refraction caustic, a highly degenerate caustic that exists for certain hexagonal crystals, exhibit a thermal conductivity that diverges logarithmically on approaching the caustic. For rods with axes close to the directions of the more generic fold and cusp caustics, the conductivity remains finite, but displays singular behavior with a 1/2- or 1/3-power law falloff with angular deviation from the caustic. Moreover, in the direction of a fold caustic, the Casimir conduction is not necessarily a maximum. Numerical results are presented for zinc, with the quasi-transverse branch providing examples of the external conical refraction and fold caustics, and in a certain sense, also the cusp caustic.
\end{abstract}

Keywords: phonon transport; thermal conduction; Casimir limit, phonon focusing 


\section{Introduction}

The Casimir model [1] for boundary-limited thermal conduction has enjoyed considerable success over the years in accounting for the thermal conductivities of dielectric rods at low temperatures, when boundary scattering of thermal phonons dominates over bulk scattering, and

the effective phonon mean free path $\bar{l}$ is controlled by the lateral dimensions of the rod. The model leads to an expression for the conductivity of a rod similar to the familiar kinetic theory result for bulk thermal conductivity

$$
\kappa=\frac{1}{3} C_{V} \bar{v} \bar{l},
$$

where $\bar{v}$ is a suitably averaged phonon velocity, and $C_{V}$ is the specific heat at constant volume. Well below the Debye temperature, since $\kappa \propto C_{V}$, the conductivity falls off as the cube of the absolute temperature $T$, a result that has been confirmed in numerous measurements, see e.g. Refs. [2-4] In recent years the main focus of attention has shifted to the burgeoning field of nanoscale thermal transport [5-7], where even at room temperature the boundary scattering of phonons can play a significant role. Studies of thermal conduction in nanowires and other nanostructures [5-13] have stimulated renewed interest in boundary-limited thermal conductivity at low temperatures [14-16].

The important influence of elastic anisotropy on thermal conduction in the Casimir regime was first recognized in the seminal paper by McCurdy, Maris and Elbaum [17]. In an elastically anisotropic medium the acoustic slowness surface, $S(\mathbf{s})=0$, i.e. the surface representing the directional dependence of the phonon slowness $\mathbf{s}=\mathbf{k}(\omega) / \omega$, where $\mathbf{k}$ the wave vector and $\omega$ the angular frequency, is non-spherical. As a consequence, the phonon group or ray velocity $\mathbf{V}=\nabla_{\mathbf{k}} \omega(\mathbf{k})$, which is normal to the slowness surface, is not in general parallel to the wave vector. This gives rise to the phenomenon of phonon focusing, whereby the ray vectors associated with a uniform distribution of phonon slownesses are strongly concentrated in directions normal to regions of the slowness surface where the curvature is small. The resulting phonon flux intensity from a point source is proportional to the Maris phonon enhancement factor [18-20]

$$
M=1 / s^{3} V|K| \text {, }
$$

where $K=4 \alpha \beta$ is the Gaussian curvature and $2 \alpha$ and $2 \beta$ are the principal curvatures of the slowness surface. A point on the slowness surface where one of the principal curvatures passes through zero on changing sign maps onto a caustic where $M=\infty$. Phonon-focusing causticssharp maxima in intensity for certain directions corresponding to zero Gaussian curvature of the slowness surface — are indeed observed in many measured phonon images.[21,22]

It might seem plausible that for a rod oriented in such a focusing direction, the Casimir conductivity should be higher than elsewhere, but this is not invariably the case, as we show below. The study [17] and subsequent works [23-29] reported numerical calculations of directionally dependent conductivities in a number of crystals of various symmetries and shapes of cross section, and experimental work [30-32] has provided evidence in support of the predictions. However, a clear physical understanding of the role of phonon-focusing caustics in Casimir conduction has hitherto been missing. What happens if the rod axis lies exactly in, or very close to the direction of a caustic? Will the thermal conductivity of an infinite rod oriented 
in a caustic direction remain finite? Will we observe different behavior for different types of caustics? Addressing these questions constitutes the substance of this paper.

We give attention to the three most common phonon-focusing caustics: (a) the line or fold caustic, which is to be found in the direction of a sharp fold in the acoustic wave surface, i.e. the surface representing the directional dependence of the group velocity, (b) the cusp caustic where two line caustics meet to form a cusp, with the cusp separating an inner region where locally the wave surface is triplicated, from the outer region where it is comprised of a single sheet, and (c) the external conical refraction caustic, which is displayed by certain hexagonal crystals, for which there is a cone of slowness vectors, all of which map onto the same group velocity vector pointing along the principal axis, and being the apex of a cone in the wave surface.

We establish that the conductivity of a long rod exhibits singular behavior when oriented in the vicinity of a phonon caustic, and identify the specific types of singular behavior for the three caustic types mentioned above. This singular behavior is determined by the local geometry of the slowness surface associated with the caustic. Superimposed on this singular component of $\kappa$, is a smoothly varying background which all phonons with a component of group velocity parallel to the rod axis contribute to, and which can therefore be regarded as a property of the global geometry of the slowness surface. For illustrative purposes we present the calculated directionally dependent effective phonon mean free path of a hexagonal zinc crystal rod, which provides examples of the three types of singularities. As expected, these singular features are less pronounced than in ballistic transport from a point source in a bulk solid. We find that the Casimir conductivity of an infinite rod oriented in the direction of a fold or cusp caustic remains finite. Surprisingly, the Casimir conduction is not necessarily even a maximum in the direction of a fold caustic. On the other hand, the thermal conductivity of an infinite rod oriented near the external refraction caustic diverges logarithmically on approach to the caustic direction. Exactly in the direction of the external conical refraction caustic, the Casimir conductivity becomes infinite, implying that in practice the conductivity will be limited either by a finite length of the rod or by remnant bulk scattering.

\section{Evaluation of Casimir Model for Anisotropic Media}

We follow a procedure established by McCurdy et al. [17] (their equations 5, 11 and 12) for calculating the thermal conductivity $\kappa$ of an infinitely long anisotropic solid cylinder of radius $R$ much larger than the dominant phonon wavelength, i.e., the Casimir conductivity. It assumes the absence of bulk scattering, perfectly diffuse scattering at the surface, and cross section isotherms. At temperatures much lower than the Debye temperature it renders the formula

$$
\kappa=\frac{4 R k_{B}}{45}\left(\frac{k_{B} T}{\hbar}\right)^{3} \sum_{j=1}^{3} \Lambda_{j} ; \Lambda_{j}=\int_{4 \pi} \frac{s_{j}^{3} V_{j \|}^{2} d \Omega}{\left|V_{j \perp}\right|},
$$

where the sum is over the three acoustic branches, $k_{B}$ is Boltzmann's constant and $\hbar$ is Planck's constant, $V_{j \|}$ is the phonon group velocity component parallel to the axis of the rod and $V_{j \perp}$ the component perpendicular to the axis. The integral is over all directions of the slowness $\mathbf{s}_{j}, d \Omega$ being the solid angle element in which the slowness vector lies. Representing the thermal conductivity by Eq. (1), the effective phonon mean free path, normalized to the diameter of the rod, is in turn given by [17] 
$\ell=\bar{\ell} / 2 R=\frac{1}{3 \pi^{2}<s^{2}>} \sum_{j=1}^{3} \Lambda_{j}$

with $\bar{v}$ interpreted as $\left\langle s^{2}\right\rangle /\left\langle s^{3}\right\rangle$, where the averages are taken over all directions and for the three acoustic branches. $\ell$ is unity in the case of an isotropic solid, but varies with direction for an anisotropic solid. In highlighting the effects of phonon focusing on Casimir conduction, there can be advantage in displaying the variation in direction of $\ell$ rather than $\kappa$.

The issue of partial specularity has been examined both from a theoretical [17] and experimental [4] point of view, and evidence points to an increase in specularity and hence reduction in diffuse scattering as the temperature is lowered and the dominant phonon wavelength increases. There is also evidence for an increase in specularity for phonons incident on the surface at grazing angles. Detailed models have been proposed for the influence of surface roughness on scattering [13]. The influence of the shape of the cross section of the rod has also been examined by various authors, and attention has also been given to finite length to lateral dimension aspect ratio. While recognizing the validity of all these concerns, the aim of the present paper is to specifically highlight the influence of elastic anisotropy, through phonon focusing, on boundary-limited thermal conduction. It serves our purposes under the circumstances to treat all other factors in as simple a manner as possible, i.e. by adopting the assumptions enunciated in the previous paragraph.

Inspection of Eq. (3) reveals that important contributions to the boundary-limited thermal conductivity in an infinitely long rod oriented in a particular direction in an anisotropic medium come from phonons of each branch for which $\left|V_{j \perp}\right|<<\left|V_{j \|}\right|$, i.e. phonons whose group velocities lie close to the axis of the rod. These are associated with portions of the slowness surface oriented normal to the axis of the rod. We proceed to calculate what this contribution is for a small angular cone of phonon slowness vectors of a particular branch (the branch index $j$ is henceforth suppressed) for which locally the slowness surface equation can be approximated by a low order polynomial expression of the form [33]

$$
S(\mathbf{s})=\left(s_{3}-s_{30}\right)+\left\{\alpha\left(s_{1}-s_{10}\right)^{2}+\beta\left(s_{2}-s_{20}\right)^{2}+\ldots . .\right\}=\hat{s}_{3}+\left\{\alpha \hat{s}_{1}^{2}+\beta \hat{s}_{2}^{2}+\ldots . .\right\}=0,
$$

where $\mathbf{s}_{0}=\left(s_{10}, s_{20} s_{30}\right)$ is the point on the slowness surface where the normal, points exactly along the rod axis. We will label this the $s_{3}$ direction, and take $s_{1}$ and $s_{2}$ to be located along the directions of the principal curvatures $2 \alpha$ and $2 \beta$ of the slowness surface at $\mathbf{s}_{0}$. For elliptic points on the slowness surface ( $\alpha$ and $\beta$ of the same sign) and hyperbolic points ( $\alpha$ and $\beta$ opposite in sign) it is sufficient to truncate the expansion in (5) after quadratic terms. The coefficients $\alpha$ and $\beta$ have dimensions of inverse slowness and it will be convenient to express results below in terms of the dimensionless quantities $\tilde{\alpha}=\alpha s_{0}$ and $\tilde{\beta}=\beta s_{0}$, and likewise we will employ the dimensionless slownesses $\tilde{s}_{1}=\hat{s}_{1} / s_{0}$, and $\tilde{s}_{2}=\hat{s}_{2} / s_{0}$. For parabolic points, where one of the principal curvatures is zero, the expansion (5) needs to be extended to higher order, as we show later.

For any given neighboring point on the slowness surface $\mathbf{s}=\mathbf{s}_{0}+\hat{\mathbf{s}}$, the associated group velocity is given by [34] 


$$
\mathbf{V}=\nabla_{\mathbf{k}} \omega(\mathbf{k})=\frac{\nabla_{\mathbf{s}} S(\mathbf{s})}{\mathbf{s} . \nabla_{\mathbf{s}} S(\mathbf{s})}
$$

yielding $\mathbf{V}_{0}=\left(0,0,1 / s_{30}\right)$ for the point $\mathbf{s}_{0}$. The solid angle element in the evaluation of (3) and (4) can be expressed as

$$
d \Omega=\frac{d \hat{s}_{1} d \hat{s}_{2}}{\left|\mathbf{s}_{0}\right|^{2}} \cos \theta=\frac{d \tilde{s}_{1} d \tilde{s}_{2} s_{30}}{s_{0}}
$$

where $\theta$ is the angle between the slowness and group velocity vectors. To highlight the contribution to $\kappa$ of phonons with slowness vectors lying within a cone of small solid angle $\delta \Omega$ around $\mathbf{s}_{0}$, within which there is little relative variation in $\alpha$ and $\beta$, we evaluate the dimensionless quantity

$\Xi=\frac{\Lambda}{s_{0}^{2}}=\frac{2}{s_{0}^{2}} \int_{\delta \Omega} d \Omega \frac{s_{0}^{3} V_{3}^{2}}{\sqrt{V_{1}^{2}+V_{2}^{2}}}=2 \int_{\delta \Omega} \frac{d \tilde{s}_{1} d \tilde{s}_{2} s_{30} V_{3}^{2}}{\sqrt{V_{1}^{2}+V_{2}^{2}}}$.

The factor of 2 has been inserted because modes around $\mathbf{s}_{0}$ and $-\mathbf{s}_{0}$ contribute equally to $\Xi$.

\subsection{Elliptic and Hyperbolic Points on Slowness Surface}

For elliptic and hyperbolic points on the slowness surface, inserting (5) into (6) for neighboring points yields

$$
\mathbf{V}=\frac{\left(2 \alpha \hat{s}_{1}, 2 \beta \hat{s}_{2}, 1\right)}{\left\{s_{30}+2 \alpha s_{10} \hat{s}_{1}+2 \beta s_{20} \hat{s}_{2}+\alpha \hat{s}_{1}^{2}+\beta \hat{s}_{2}^{2}\right\}} \approx \frac{1}{s_{30}}\left(2 \alpha \hat{s}_{1}, 2 \beta \hat{s}_{2}, 1\right)=\frac{1}{s_{30}}\left(2 \tilde{\alpha} \tilde{s}_{1}, 2 \tilde{\beta} \tilde{s}_{2}, 1\right),
$$

to first order in the slownesses. Substituting (9) into (8) one obtains

$$
\Xi=2 \int_{\Omega \Omega} \frac{d \tilde{s}_{1} d \tilde{s}_{2}}{\sqrt{4 \tilde{\alpha}^{2} \tilde{s}_{1}^{2}+4 \tilde{\beta}^{2} \tilde{s}_{2}^{2}}} .
$$

With change of integration variables to $x=2 \tilde{\alpha} \tilde{s}_{1}$ and $y=2 \tilde{\beta} \tilde{s}_{2}$, and assuming a square integration domain of side $2 x_{\max }$, within which there is little relative variation in $\tilde{\alpha}$ and $\tilde{\beta}$ we obtain

$\Xi=\frac{16 \ln (1+\sqrt{2}) x_{\max }}{s_{0}^{2}|K|}$

This result is reminiscent of the Maris phonon enhancement factor given by Eq. (2), except very importantly for the presence of the factor $x_{\max }$. So while one might be inclined to infer that the Casimir conductivity should be relatively large for rods oriented in high phonon focusing directions where $|K|$ is small and $M$ is large, this has to be tempered by the fact that such directions are often near phonon-focusing caustics where one of the inverse curvatures is changing very rapidly, and $x_{\max }$ needs to be taken very small. The only unambiguous conclusions one can thus draw from this analysis for generic directions, that is directions well away from phonon caustics, are that (i) $\kappa$ is finite and a smoothly varying function of direction, and (ii) all phonons of all three branches need to be considered in calculating $\kappa$, i.e. $\kappa$ is dependent on the global rather than just the local geometry of the slowness surface.

\subsection{Parabolic Points on Slowness Surface Associated with a Fold Caustic}


At parabolic points on the slowness surface, i.e. points where one of the principal curvatures, say $\beta$, is zero, the expansion (5) needs to be extended to higher order to read $[33,35,36]$

$S(\mathbf{s})=\left(s_{3}-s_{30}\right)+\left\{\alpha\left(s_{1}-s_{10}\right)^{2}+\gamma\left(s_{2}-s_{20}\right)^{3}\right\}=\hat{s}_{3}+\left\{\alpha \hat{s}_{1}^{2}+\gamma \hat{s}_{2}^{3}\right\}=0$.

Without loss of generality we may assume $\alpha$ and $\gamma$ to be positive. To lowest order in $\tilde{s}_{1}, \tilde{s}_{2}$ we now have that

$\mathbf{V} \approx \frac{1}{s_{30}}\left(2 \tilde{\alpha} \tilde{s}_{1}, 3 \tilde{\gamma} \tilde{s}_{2}^{2}, 1\right)$

where $\tilde{\gamma}=\gamma s_{0}^{2}$. Notice that with variation of $\tilde{s}_{2}$ from negative to positive values, the component $V_{2}$ decreases to zero and then increases again. Locally the wave surface, i.e. the locus of all group velocity vectors, is thus folded across $V_{2}=0$, along a line in the $\hat{1}$ direction, being duplicated for $V_{2}>0$ and non-existent beyond the fold edge. The fold edge is a line of accumulation of rays representing the fold caustic, where the Maris phonon enhancement factor $M$ is infinite.

Of interest is the behavior of $\Xi$ in the neighborhood of the fold, i.e. the variation of $\Xi$ with small angular deviation $\psi$ of the rod axis from the fold caustic. In directions of positive $\psi$, where the wave surface is doubled up, the Maris phonon enhancement factor varies as $M \sim \psi^{-1 / 2}$, diverging at the fold. Directions of negative $\psi$ lie beyond the fold edge, where $M=0$. Interestingly though, because all phonons with a non-zero component of group velocity along the rod axis contribute to the Casimir conduction, albeit heavily weighted towards phonons near the rod axis, we will see that $\Xi(\psi)$ does not drop immediately to zero beyond the fold, but instead shows a singular falloff as $\sim-|\psi|^{1 / 2}$, as the modes in the folded region contribute less and less to $\Xi(\psi)$ with increasing $|\psi|$.

With a rotation of axes about the $\hat{1}$ direction by the small angle $\psi$ to place the $V_{3}$ axis along the rod axis, we have that

$$
\mathbf{V} \approx \frac{1}{S_{30}}\left(2 \tilde{\alpha} \tilde{s}_{1}, 3 \tilde{\gamma} \tilde{s}_{2}^{2}-\psi, 1\right) \text {. }
$$

Substituting into (8) we obtain

$$
\Xi(\psi)=2 \int_{\delta \Omega} \frac{d \tilde{s}_{1} d \tilde{s}_{2}}{\sqrt{4 \tilde{\alpha}^{2} \tilde{s}_{1}^{2}+\left(3 \tilde{\gamma} \tilde{s}_{2}^{2}-\psi\right)^{2}}} .
$$

With change of integration variables to $x=2 \tilde{\alpha} \tilde{s}_{1}$ and $y=\sqrt{3 \tilde{\gamma}} \tilde{s}_{2}$, and assuming as before a square integration domain of side $2 x_{\max }$, within which there is little relative variation in $\tilde{\alpha}$ and $\tilde{\gamma}$, we obtain

$$
\Xi(\psi)=\frac{1}{\left(3 \tilde{\alpha}^{2} \tilde{\gamma}\right)^{1 / 2}} \int_{-x_{\max }}^{x_{\max }} d y \int_{-x_{\max }}^{x_{\max }} d x \frac{1}{\sqrt{x^{2}+\left(y^{2}-\psi\right)^{2}}} .
$$

The integration with respect to $x$ can be done analytically yielding

$$
\Xi(\psi)=\frac{1}{\left(3 \tilde{\alpha}^{2} \tilde{\gamma}\right)^{1 / 2}} \int_{-x_{\max }}^{x_{\max }} d y\left\{\ln \left(x_{\max }+\sqrt{x_{\max }^{2}+\left(y^{2}-\psi\right)^{2}}\right)-\ln \left(-x_{\max }+\sqrt{x_{\max }^{2}+\left(y^{2}-\psi\right)^{2}}\right)\right\} .
$$


The integration with respect to $y$ is readily done numerically for any given value of $x_{\max }$. Figure 1 shows a calculated plot of $\Xi(\psi)$ for $\left(3 \tilde{\alpha}^{2} \tilde{\gamma}\right)^{1 / 2}=1$ and $x_{\max }=0.25$, and also plotted is the expression

$\Xi(\psi)_{f i t}=A-B|\psi|^{1 / 2} H(-\psi)-C \psi$,

where $H(\psi)$ is the unit step function and $A=4.082, B=12.57, C=16.0$. The two curves are virtually indistinguishable. The coefficients $A$ and $C$ depend on the size and shape of the integration domain, and are thus conditioned by the global geometry of the slowness surface. On the other hand, for $|\psi|<<x_{\max }<<1, B$ is independent of $x_{\max }$, being equal, as we show below, to $4 \pi /\left(3 \tilde{\alpha}^{2} \tilde{\gamma}\right)^{1 / 2}$. It is determined entirely by the local geometry of the slowness surface. $B$ determines the singular behavior of the Casimir conduction with a falloff as $-|\psi|^{1 / 2}$ beyond the line caustic. Surprisingly, in view of the divergence of the Maris phonon intensity factor towards the caustic from inside the folded region, for rod axes that intersect the folded region of the wave surface $\Xi$ displays no singular behavior, only linear variation with direction. By extending the integration to cover the entire slowness surface, $A$ and $C$ change, but $B$ is unaffected.

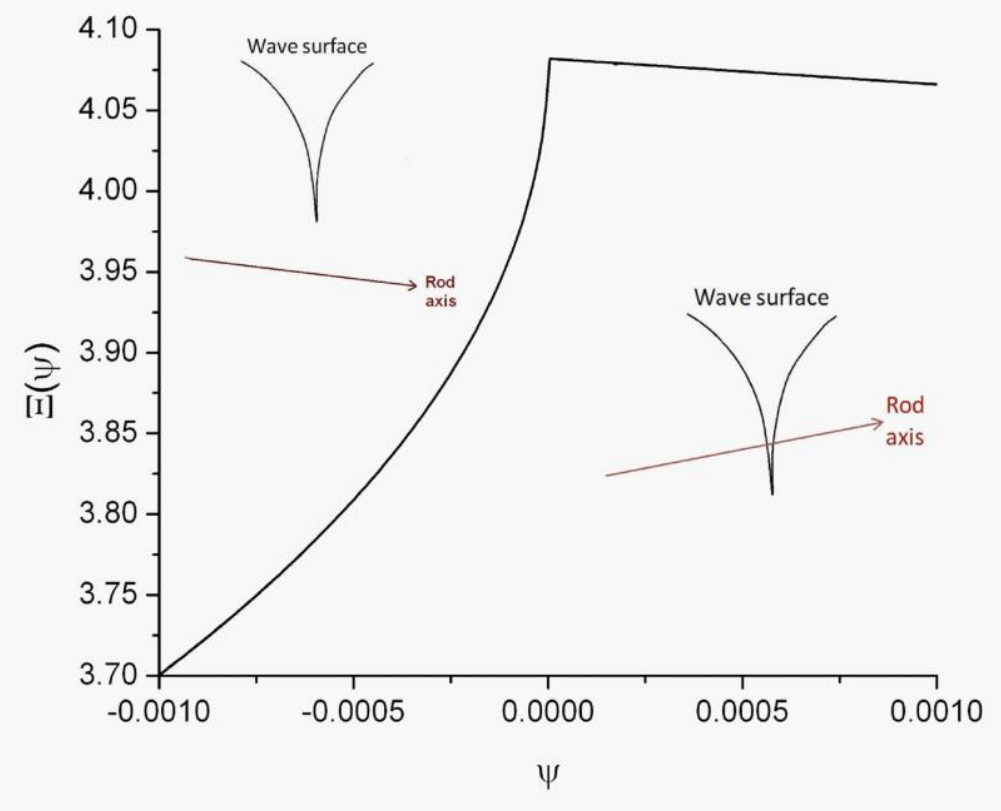

Figure 1. $\Xi(\psi)$ for the fold caustic.

The singular behavior of $\Xi(\psi)$ for negative $\psi$ can be accounted for by evaluating the integral in (17) analytically in the limit $|\psi|<<x_{\max }<<1$. The second of the two logarithms in (17) is the source of this singular behavior, with the first term yielding a contribution that varies smoothly around $\psi=0$. Changing the sign in front of the first logarithm makes no difference to the singular behavior, but it does have a great advantage. For, by now combining the two logarithms as the log of a product, this leads to the rationalization of the argument of the logarithm as the difference between two squares, greatly simplifying the evaluation of the integral. The singular part of $\Xi(\psi)$ for $\psi<0$ thereby takes the form 
$\Xi(\psi)_{\text {singular }}=-\frac{1}{\left(3 \tilde{\alpha}^{2} \tilde{\gamma}\right)^{1 / 2}} \int_{-x_{\max }}^{x_{\max }} d y \ln \left\{\left(\sqrt{x_{\max }^{2}+\left(y^{2}+|\psi|\right)^{2}}\right)^{2}-x_{\max }^{2}\right\}=-\frac{2}{\left(3 \tilde{\alpha}^{2} \tilde{\gamma}\right)^{1 / 2}} \int_{-x_{\max }}^{x_{\max }} d y \ln \left(y^{2}+|\psi|\right) \cdot(1$

This is a standard integral, which in the limit of $|\psi| / x_{\max }^{2}<<1$ is equal to $2 \pi \sqrt{|\psi|} / x_{\max }+2|\psi| / x_{\max }^{2}-4$, the second and third terms being non-singular. Thus, we have proven that

$\Xi(\psi)_{\text {singular }}=\frac{-4 \pi}{\left(3 \tilde{\alpha}^{2} \tilde{\gamma}\right)^{1 / 2}}|\psi|^{1 / 2} H(-\psi)$,

independent of the value of $x_{\max }$, and thus determined uniquely by the local geometry of the slowness surface.

\subsection{Parabolic Points on Slowness Surface Associated with a Cusp Caustic}

At isolated points on a parabolic line on the slowness surface, the direction of vanishing principal curvature may be parallel to the parabolic line. [37] These map onto cuspidal points in the wave surface where two fold edges meet, and there is a cusp caustic, see Figure 2. Within the cuspidal region, locally there are three sheets to the folded wave surface, and outside there is one. Taking the principal curvatures $\beta$ to be zero, the expansion (5) reads [33,35]

$$
S(\mathbf{s})=\left(s_{3}-s_{30}\right)+\left\{\alpha\left(s_{1}-s_{10}\right)^{2}+\xi\left(s_{1}-s_{10}\right)\left(s_{2}-s_{20}\right)^{2}+\varepsilon\left(s_{2}-s_{20}\right)^{4}\right\}=\hat{s}_{3}+\left\{\alpha \hat{s}_{1}^{2}+\xi \hat{s}_{1} \hat{s}_{2}^{2}+\varepsilon \hat{s}_{2}^{4}\right\}=0,
$$

where without loss of generality we can assume $\alpha$ and $\xi$ to be positive. In order to simplify the algebra in the presentation, the derivations in the following will be shown for $\varepsilon=0$, but we will provide end results for nonzero $\varepsilon \hat{S}_{2}^{4}$. This term is formally part of the generating function for the cusp caustic, and its main effect is to alter the aspect ratio of the cusp, and for $4 \alpha \varepsilon / \xi^{2}>1$ to reverse its direction.

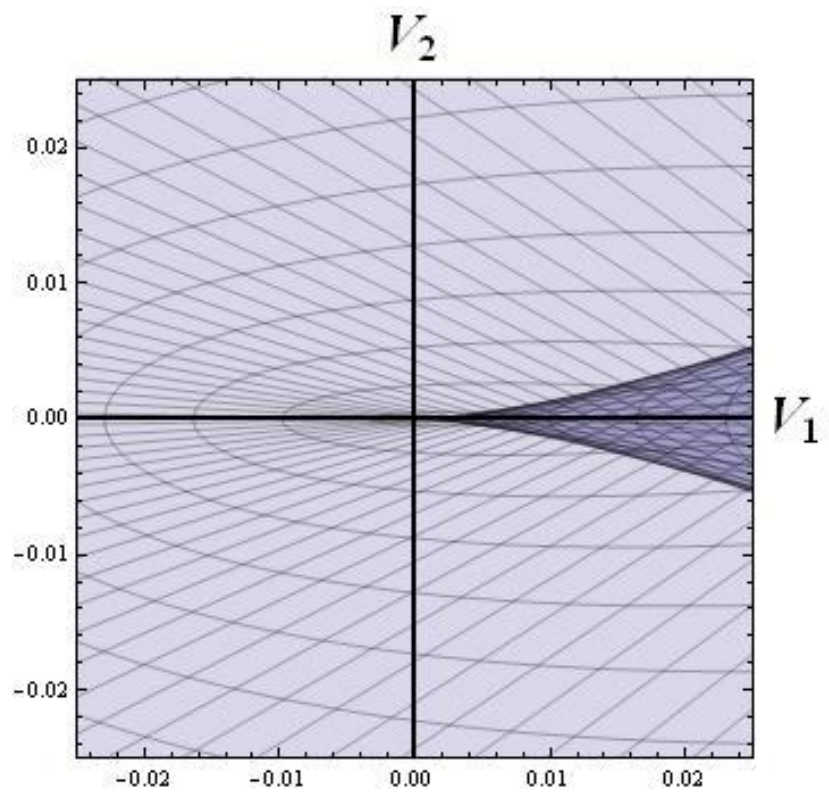

Figure 2. The cusp caustic, showing contours of constant $\tilde{s}_{2}$ (the straight lines) and constant $\tilde{s}_{1}$ (the curves) on a projection of the wave surface. The caustic is envelope to the $\tilde{s}_{2}$ contours and to the positive $\tilde{s}_{1}$ contours (parabolas located inside of the cuspidal region). 
To lowest order in $\hat{s}_{1}, \hat{s}_{2}$ the group velocity in this case is given by

$\mathbf{V} \approx \frac{1}{s_{30}}\left(2 \tilde{\alpha} \tilde{s}_{1}+\tilde{\xi} \tilde{s}_{2}^{2}, 2 \tilde{\xi} \tilde{s}_{1} \tilde{s}_{2}, 1\right)$

where $\tilde{\xi}=\xi s_{0}^{2}$, and for later use $\tilde{\varepsilon}=\varepsilon s_{0}^{3}$.

\subsubsection{Deviation of the rod axis at right angles to the pointing direction of the cusp}

For deviation of the rod axis in the $\hat{2}$ direction, i.e. perpendicular to the direction in which the cusp is pointing, we affect a rotation of the axes by small angle $\psi$ about the $\hat{1}$ direction to place the $\hat{2}$ direction along the rod axis, obtaining

$\mathbf{V} \approx \frac{1}{s_{30}}\left(2 \tilde{\alpha} \tilde{s}_{1}+\tilde{\xi} \tilde{s}_{2}^{2}, 2 \tilde{\xi} \tilde{s}_{1} \tilde{s}_{2}-\psi, 1\right)$,

and substituting into (8) we obtain

$\Xi(\psi)=\int_{\infty 2} \frac{d \tilde{s}_{1} d \tilde{s}_{2}}{\sqrt{\left(\tilde{\alpha} \tilde{s}_{1}+\tilde{\xi} \tilde{s}_{2}^{2} / 2\right)^{2}+\left(\tilde{\xi} \tilde{s}_{1} \tilde{s}_{2}-\psi / 2\right)^{2}}}$.

With change of integration variables to $x=(\tilde{\xi} / \tilde{\alpha}) \tilde{s}_{1}$ and $y=(\tilde{\xi} / \tilde{\alpha}) \tilde{s}_{2}$, and assuming as before a square integration domain of side $2 x_{\max }$, one has

$\Xi(\psi)=\frac{1}{\tilde{\xi}} \int_{-x_{\max }}^{x_{\max }} d y \int_{-x_{\max }}^{x_{\max }} d x \frac{1}{\sqrt{\left(x+y^{2} / 2\right)^{2}+(x y-\tilde{\psi} / 2)^{2}}}$,

where $\tilde{\psi}=\tilde{\xi} \psi / \tilde{\alpha}^{2}$. Figure 3 shows the result obtained by doing the integration in (25) numerically for $x_{\max }=y_{\max }=0.25$ and $\tilde{\alpha}=0.1$ and $\tilde{\xi}=0.1$ compared with analytical fit to the expression

$\Xi(\psi)_{f i t}=A-B|\psi|^{1 / 3}$,

with $A=71.3$ and $B=234.4$. The two curves are virtually indistinguishable. The coefficient $A$ depends on the size and shape of the integration domain and is thus a function of the global geometry of the slowness surface. On the other hand, analytic integration of (25), in the limit $|\psi|<<x_{\max }<<1$, shows that $B=2 \sqrt{3} \pi /(\tilde{\alpha} \tilde{\xi})^{2 / 3}=10.88 /(\tilde{\alpha} \tilde{\xi})^{2 / 3}$ and is independent of $x_{\max }$, and is thus determined entirely by the local geometry of the slowness surface. The proof of this follows along similar lines to that of the singular behavior for the cusp caustic. B describes the singular behavior of the Casimir conduction near the cusp in the perpendicular direction, falling off symmetrically as $\sim-|\psi|^{1 / 3}$, as the modes in the cuspidal region contribute less and less. By extending the integration to cover the entire slowness surface, $A$ changes, but $B$ is unaffected. The symmetry of the cusp requires that higher order terms should be powers of $|\psi|$ rather than of $\psi$. With the inclusion of the $\varepsilon \hat{s}_{2}^{4}$ term in the generating function for the cusp caustic the singular behavior of $\Xi$ is given by

$\Xi(\psi)_{\text {singular }}=\frac{2 \sqrt{3} \pi}{\left(\tilde{\alpha}^{2}\left|\tilde{\xi}^{2}-4 \tilde{\alpha} \tilde{\varepsilon}\right|\right)^{1 / 3}}|\psi|^{1 / 3}$. 


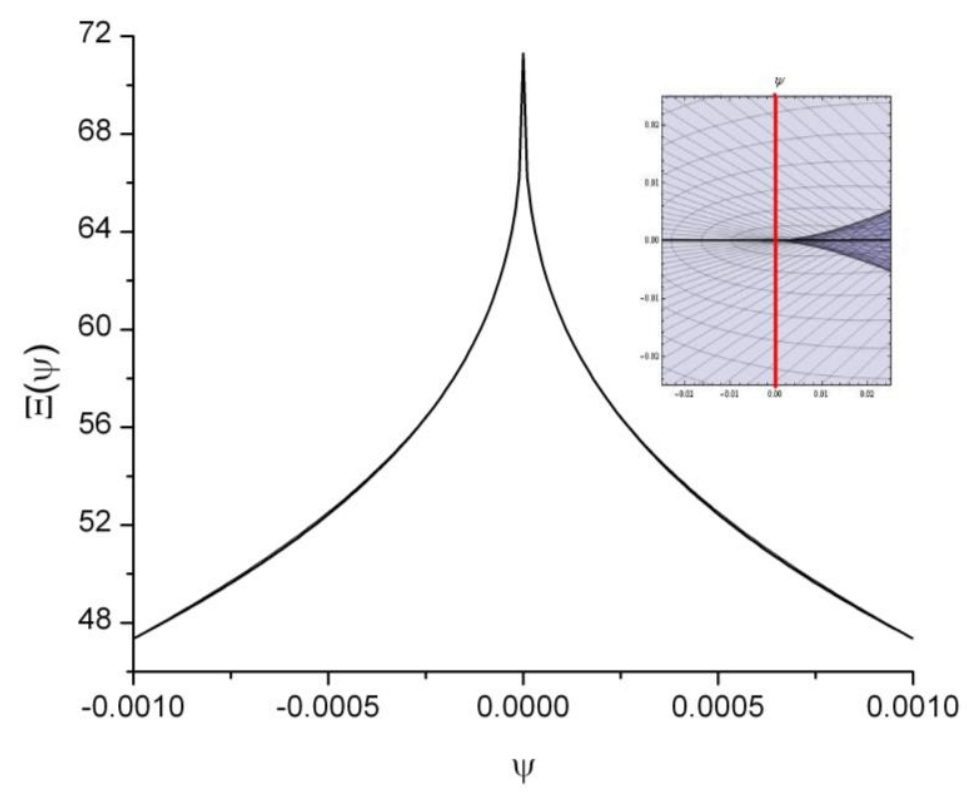

Figure 3. $\Xi(\psi)$ for the cusp caustic at right angles to the pointing direction of the cusp.

\subsubsection{Deviation of the rod axis in the pointing direction of the cusp}

For deviation of the rod axis in the $\hat{1}$ direction, i.e. in the direction in which the cusp is pointing, we effect a rotation of the axes by small angle $\psi$ about the $\hat{2}$ direction, obtaining $\mathbf{V} \approx \frac{1}{s_{30}}\left(2 \tilde{\alpha} \tilde{s}_{1}+\tilde{\xi}_{\tilde{s}_{2}}^{2}-\psi, 2 \tilde{\xi}_{\tilde{s}_{1}} \tilde{s}_{2}, 1\right)$.

Substituting into (8) and effecting the same change of integration variables to $x, y$ as before we arrive at

$\Xi(\psi)=\frac{1}{\tilde{\xi}} \int_{-x_{\max }}^{x_{\max }} d y \int_{-x_{\max }}^{x_{\max }} d x \frac{1}{\sqrt{\left(x+y^{2} / 2-\tilde{\psi} / 2\right)^{2}+(x y)^{2}}}$,

where $\tilde{\psi}=\tilde{\xi} \psi / \tilde{\alpha}^{2}$. Figure 4 shows the result obtained by doing the integration in (29) numerically for $x_{\max }=y_{\max }=0.25$ and $\tilde{\alpha}=0.1$ and $\tilde{\xi}=0.1$, compared with analytical fit to the expression

$\Xi(\psi)_{f t t}=A-B|\psi|^{1 / 2} H(-\psi)-C \psi$,

and $A=71.25, B=379, C=1660$. The two curves are virtually indistinguishable. While $A$ and $C$ depend on the size and shape of the integration domain, and are thus functions of the global geometry of the slowness surface, $B$ is independent of $x_{\max }$ and for $|\psi|<<x_{\max }<<1$ is equal to $4 \pi /\left(\tilde{\alpha}^{2} \tilde{\xi}\right)^{1 / 2}$. This quantity determines the singular behavior of the Casimir conduction for deviations from the cusp in the direction the cusp is pointing, falling off as $\sim-|\psi|^{1 / 2}$ outside the cusp, as the modes in the cuspidal region contribute less and less. Inside the cusp $\Xi$ is nonsingular. Thus the singular behavior is determined entirely by the local geometry of the slowness surface, and mimics that of the fold caustic. With the inclusion of the $\varepsilon \hat{s}_{2}^{4}$ term in the generating 
function for the cusp caustic, the direction of the cusp depends on the sign of $1-4 \tilde{\alpha} \tilde{\varepsilon} / \tilde{\xi}^{2}$, and the singular behavior of $\Xi$ is given by

$\Xi(\psi)_{\text {singular }}=-\frac{4 \pi}{\left(\tilde{\alpha}^{2} \tilde{\xi}\left|1-4 \tilde{\alpha} \tilde{\varepsilon} / \tilde{\xi}^{2}\right|\right)^{1 / 2}}|\psi|^{1 / 2} H\left(-\psi \operatorname{sgn}\left(1-4 \tilde{\alpha} \tilde{\varepsilon} / \tilde{\xi}^{2}\right)\right)$.

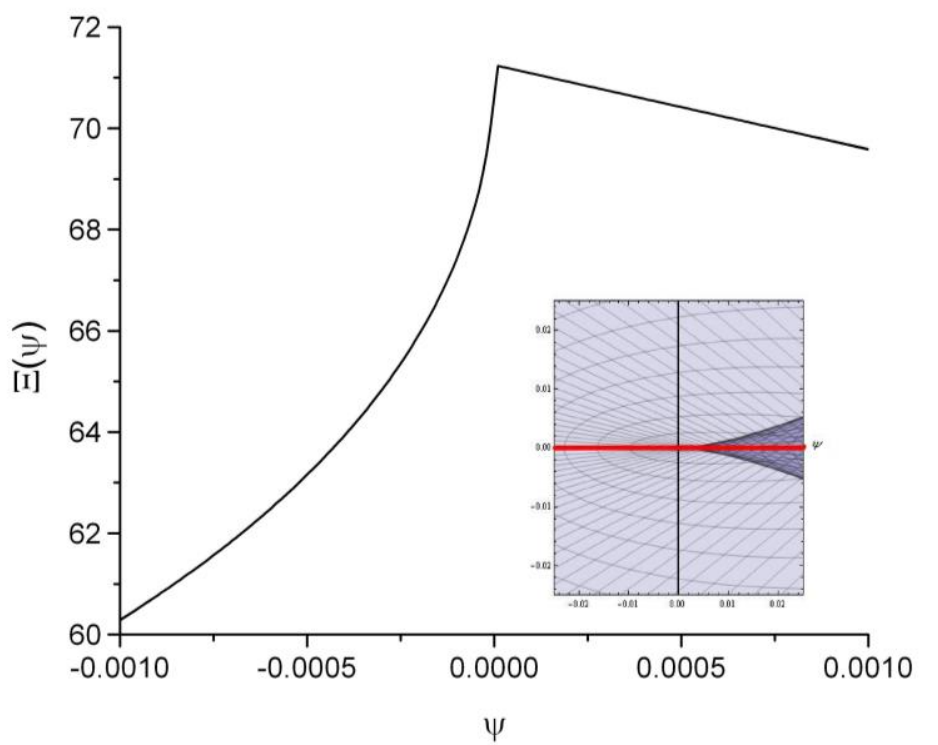

Figure 4. $\Xi(\psi)$ for the cusp caustic in the pointing direction of the cusp.

\subsection{External Conical Refraction Caustic in Transversely Isotropic Media}

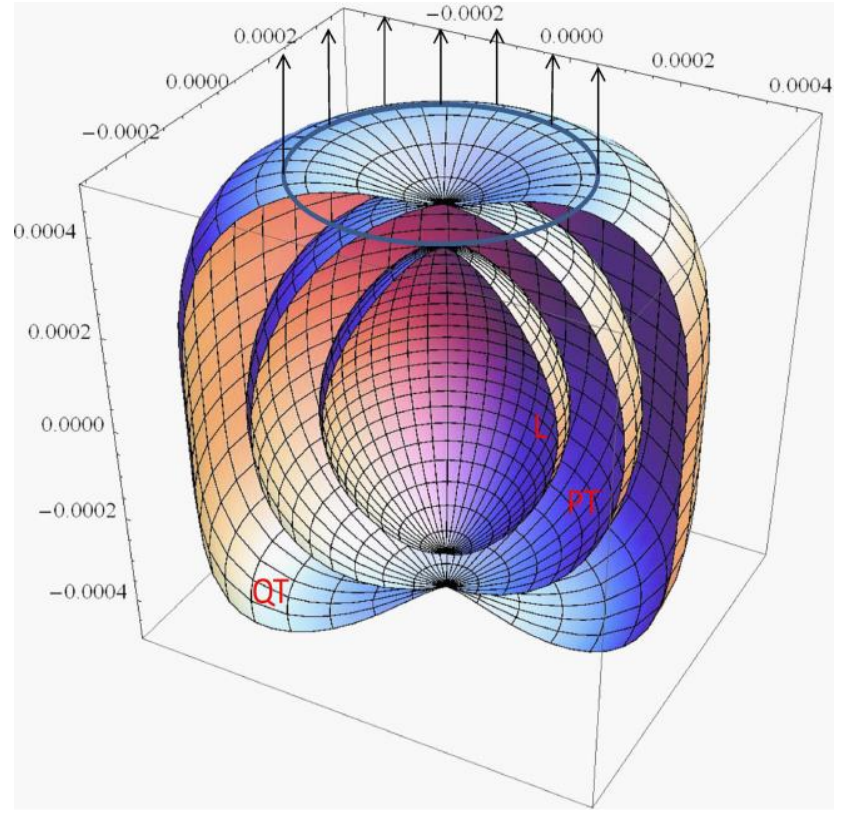

(a)

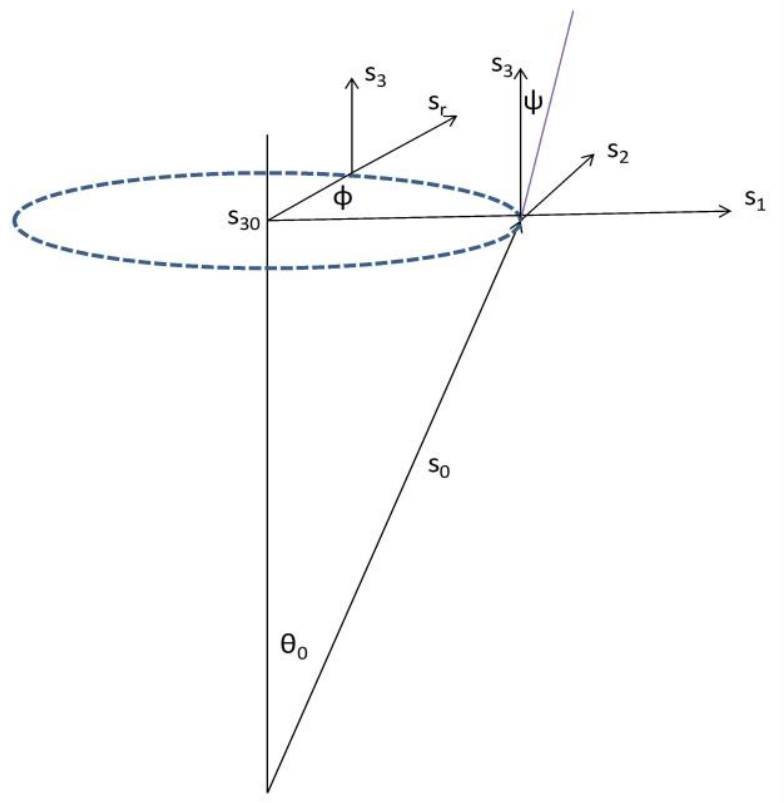

(b)

Figure 5. (a) Slowness surface for a zinc crystal (acoustically transversely isotropic) showing the Mexican hat shape of the QT sheet near the zonal axis, (b) coordinate system for the integration to obtain $\Xi(\psi)$. 
As regards their linear elastic properties, hexagonal crystals are transversely isotropic, with their acoustic surfaces exhibiting full rotational symmetry about the zonal [001] axis. One sheet of the slowness surface pertains to pure transverse (PT) modes and the other two sheets are labeled the longitudinal (L) and quasi-transverse (QT) sheets, see Fig. 5(a) which depicts the slowness surface of zinc. In the case of crystals such as zinc, for which the elastic constants satisfy the inequality $[33,38]$

$$
\frac{\left(C_{13}+C_{44}\right)^{2}}{\left[C_{11}\left(C_{33}-C_{44}\right)\right]}>1 \text {, }
$$

near the zonal axis the two portions of the QT sheet are shaped like a Mexican hat. For the upper portion, it is an inverted hat, being concave near the centre. Further out there is a circle of points (the "rim") where it starts to slope downwards, and where all rays point along the zonal axis. This is a parabolic line on the slowness surface for which the direction of vanishing principal curvature is tangential to the circle. The intense focusing of rays along the zonal axis is known as the external conical refraction caustic.[39,40] This is the one generic caustic for which the Casimir conductivity formally diverges exactly in the direction of the caustic.

To establish the directional dependence of the Casimir conductivity near the zonal axis we integrate over an annulus on the slowness surface straddling the parabolic line. The angle subtended by this circle at the origin is $\theta_{0}$ (see Figure 5(b)). Near to this circle the equation for the slowness is independent of the azimuthal direction, and takes the form

$$
S(\mathbf{s})=\left(s_{3}-s_{30}\right)+\alpha\left(s_{r}-s_{r 0}\right)^{2}=\hat{s}_{3}+\alpha \hat{s}_{r}^{2}=0,
$$

for the axial and radial components of slowness. The radial and axial components of the group velocity are $\frac{1}{s_{30}} 2 \alpha \hat{s}_{r}=\frac{1}{s_{30}} 2 \tilde{\alpha} \tilde{s}_{r}$ and $\frac{1}{s_{30}}$, where $\tilde{\alpha}=\alpha s_{0}$ and $\tilde{s}_{r}=\hat{s}_{r} / s_{0}$, and the group velocity in Cartesian components for a point located at azimuthal angle $\phi$ on the slowness surface is

$$
\mathbf{V}=\frac{1}{S_{30}}\left(2 \tilde{\alpha} \tilde{s}_{r} \cos \phi, 2 \tilde{\alpha} \tilde{s}_{r} \sin \phi, 1\right) \text {. }
$$

For a rod inclined at a small angle $\psi$ to the zonal axis, we effect a rotation of the coordinate axes about the $\hat{2}$ direction by angle $\psi$ to obtain

$$
\mathbf{V} \approx \frac{1}{s_{30}}\left(2 \tilde{\alpha} \tilde{s}_{r} \cos \phi-\psi, 2 \tilde{\alpha} \tilde{s}_{r} \sin \phi, 1\right) \text {. }
$$

In the integration to obtain $\Xi$, a rectangular element of sides $d \tilde{s}_{r}$ and $\tilde{s}_{r} d \phi$ within the annulus of the slowness surface, and which subtends solid angle $d \Omega \approx \frac{s_{30} \sin \theta_{0} d \phi \cdot d \tilde{s}_{r}}{s_{0}}$ at the origin, is used. The integral takes the form:

$$
\Xi(\psi)=2 \sin \theta_{0} \int_{\Omega} \frac{d \phi d \tilde{s}_{r}}{\sqrt{\left(2 \tilde{\alpha} \tilde{s}_{r} \cos \phi-\psi\right)^{2}+\left(2 \tilde{\alpha} \tilde{s}_{r} \sin \phi\right)^{2}}} .
$$

With change of integration variable to $x=2 \tilde{\alpha} \tilde{s}_{r}$ we have

$$
\Xi(\psi)=\frac{\sin \theta_{0}}{\tilde{\alpha}} \int_{0}^{2 \pi} d \phi \int_{-x_{\max }}^{x_{\max }} \frac{d x}{\sqrt{(x \cos \phi-\psi)^{2}+(x \sin \phi)^{2}}} .
$$


Figure 6 shows the result of evaluating the integral in Eq. (37) numerically for $x_{\max }=0.1$ and small but finite $\psi$ compared with analytical fit to the expression

$$
\frac{\tilde{\alpha}}{\sin \theta_{0}} \Xi=A+B \ln (\psi)
$$

with $A=-11.55$ and $B=-4 \pi$. The two curves are virtually indistinguishable. While the value of $A$ depends on the value of $x_{\max }$ and is thus a global property of the slowness surface, $B$ is independent of $x_{\max }$. Thus, the Casimir conduction is characterized, apart from an additive constant, by a logarithmic divergence at an external conical refraction caustic, determined entirely by the local geometry of the slowness surface at the circular parabolic line.

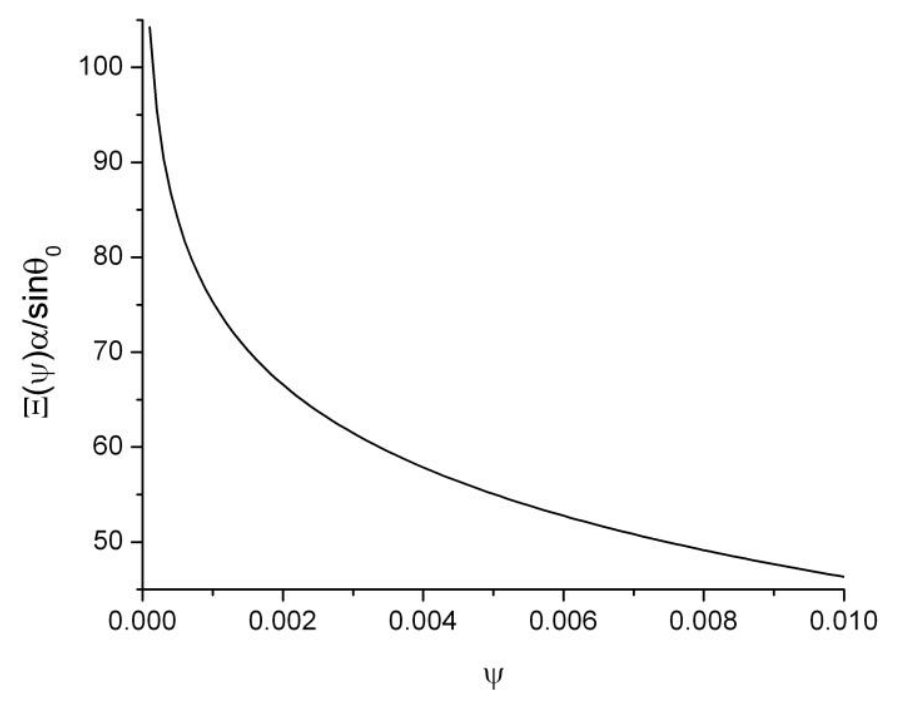

Figure 6. $\frac{\tilde{\alpha}}{\sin \theta_{0}} \Xi(\psi)$ for the external conical refraction caustic.

The singular behavior of $\Xi(\psi)$ can be accounted for by evaluating the integral in (37) analytically in the limit $0<\psi<<x_{\max }<<1$. With change of integration variable to $z=x / x_{\max }$ we have

$$
\Xi(\psi)=\frac{\sin \theta_{0}}{\tilde{\alpha}} \int_{0}^{2 \pi} d \phi \int_{-1}^{1} \frac{d z}{\sqrt{z^{2}-2 z \tilde{\psi} \cos \phi+\tilde{\psi}^{2}}} \simeq \frac{\sin \theta_{0}}{\tilde{\alpha}} \int_{0}^{2 \pi} d \phi \int_{-1}^{1} \frac{d z}{\sqrt{z^{2}-2 z \tilde{\psi} \cos \phi}},
$$

where $\tilde{\psi}=\psi / x_{\max }$. Integrating with respect to $z$ yields

$\Xi(\psi)=\frac{\sin \theta_{0}}{\tilde{\alpha}} \int_{0}^{2 \pi} d \phi\{-2 \ln \psi+$ a constant and higher order terms $\}$,

and thus the singular behavior of $\Xi$ is given by

$\Xi(\psi)_{\text {singular }}=-\frac{4 \pi \sin \theta_{0}}{\tilde{\alpha}} \ln \psi$,

independent of the value of $x_{\max }$, and dependent only on the local geometry of the slowness surface at the circular parabolic line. 


\section{Normalized Phonon Mean Free Path for a Zinc Rod}

We illustrate the results of the previous section by presenting calculations of the directional dependence of the Casimir normalized mean free path $\ell$ for a zinc rod. Normally the thermal conductivity of zinc is dominated by electrons; at temperatures well below the superconducting transition temperature, phonon-mediated thermal transport will become dominant [24], hence Casimir conductance of a zinc rod is not entirely irrelevant. However, the main reason for choosing this material for a numerical example is that it allows us to illustrate the behavior of the Casimir conductivity for all three types of caustics discussed above. Zinc is a hexagonal crystal, and is thus transversely isotropic as regards its linear elastic properties. Its slowness and wave surfaces have full rotational symmetry about the zonal $(z)$ axis. The group velocity $\mathbf{V}$ lies in the same meridian plane as the slowness $\mathbf{s}$, but in general inclined at the different angle to the zonal axis. The integration in Eq. (4) to obtain $\ell$ as a function of the inclination angle $\psi$ of the rod to the zonal axis is carried out over the polar and azimuthal angles $\theta$ and $\phi$ respectively of the slowness $\mathbf{s}$. The QT mode of zinc provides examples of the fold and external conical refraction caustic, and we will show how the behavior of $\ell$ near the equatorial plane can be interpreted as that of a slightly unfolded cusp caustic.

For a transversely isotropic medium the Christoffel slowness equation factorizes into a quadratic equation for the PT mode and a bi-quadratic for the QT and L modes. [41] For the PT mode

$$
\begin{aligned}
& S(\mathbf{s})=C_{66} s_{r}^{2}+C_{44} s_{z}^{2}-\rho=0 ; s_{r}=s \sin \theta, s_{z}=s \cos \theta, \\
& s(\theta)=\sqrt{\rho /\left(C_{66} \sin ^{2} \theta+C_{44} \cos ^{2} \theta\right)},
\end{aligned}
$$

and from (6)

$$
\mathbf{V}=\left(V_{r}, V_{z}\right)=\left(C_{66} s_{r}, C_{44} S_{z}\right) / \rho,
$$

The slownesses for the QT and L modes are given by

$$
s(\theta)=\sqrt{\rho / 2\left(B \pm \sqrt{B^{2}-4 C}\right)} \text {, }
$$

+ for the L mode and - for the QT mode. where

$$
\begin{aligned}
& B=\left(C_{11}+C_{44}\right) \sin ^{2} \theta+\left(C_{33}+C_{44}\right) \cos ^{2} \theta, \\
& C=C_{d} \sin ^{4} \theta+C_{e} \cos ^{4} \theta+C_{f} \sin ^{2} \theta \cos ^{2} \theta, \\
& C_{d}=C_{11} C_{44}, \\
& C_{e}=C_{33} C_{44}, \\
& C_{f}=C_{11} C_{33}+C_{44}^{2}-\left(C_{13}+C_{44}\right)^{2} .
\end{aligned}
$$

The group velocities are given by

$$
\mathbf{V}=\left(V_{r}, V_{z}\right)=\frac{1}{s^{2}}\left(s \sin \theta-\frac{d s}{d \theta} \cos \theta, s \cos \theta+\frac{d s}{d \theta} \sin \theta\right)
$$

In the coordinate system of the cylinder, oriented at an angle $\psi$ to the $z$ axis, the components of $\mathbf{V}$ are

$V_{3}=V_{z} \cos \psi+V_{r} \cos \phi \sin \psi$,

$V_{2}=V_{r} \sin \phi$,

$V_{1}=V_{z} \sin \psi-V_{r} \cos \phi \cos \psi$. 
With $\mathbf{V}$ expressed in terms of $\theta$ and $\phi$ and with the rod orientation angle $\psi$ as parameter, the integrations in Eqs. (3) and (4) are evaluated numerically to obtain the conductivity $\kappa$ or, more conveniently for comparison with the existing literature, the normalized mean free path $l$. The directional dependence of $l$ for zinc, obtained from Eq.(4), and based on density $\rho=7140$ $\mathrm{kg} / \mathrm{m}^{3}$ and the following elastic constants [42]: $C_{11}=165, C_{33}=61.8, C_{44}=39.6, C_{13}=50.0$, $C_{12}=31.1$ in $\mathrm{GPa}, C_{66}=\left(C_{11}-C_{12}\right) / 2$, is shown in Figure 7.

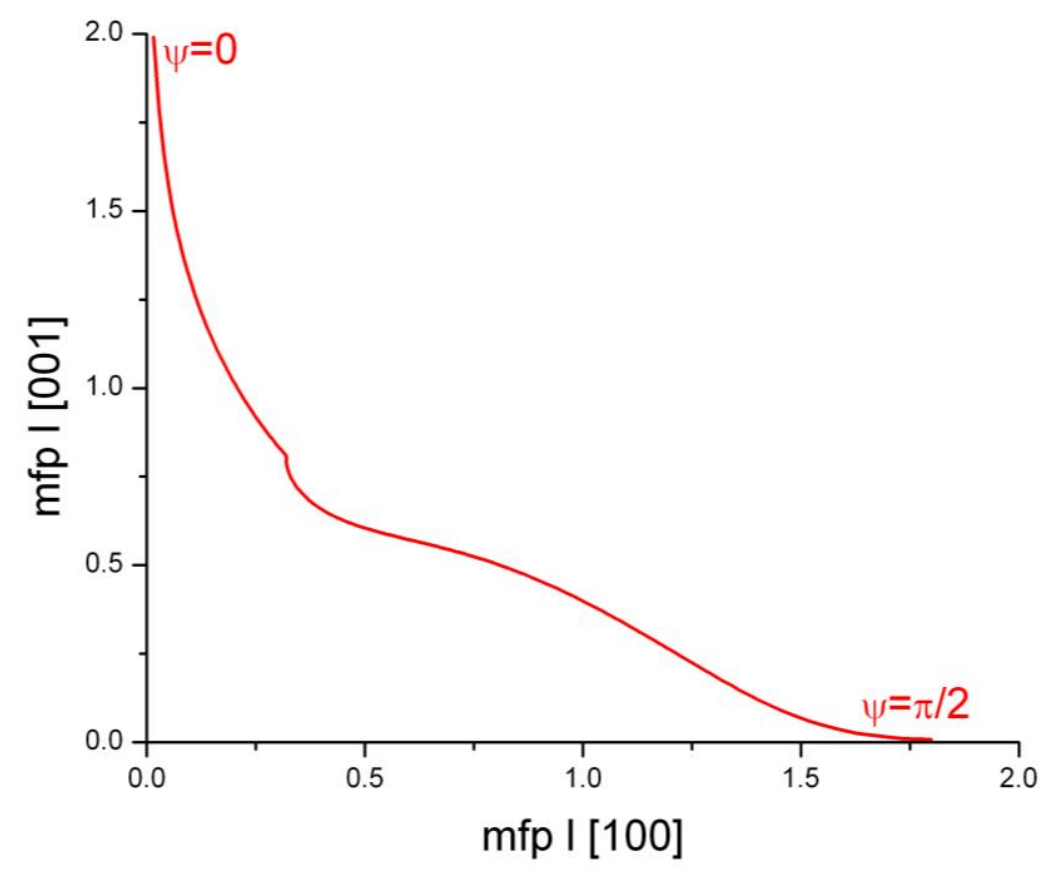

Figure 7. Variation of normalized phonon mean free path $l$ for a zinc rod with direction .

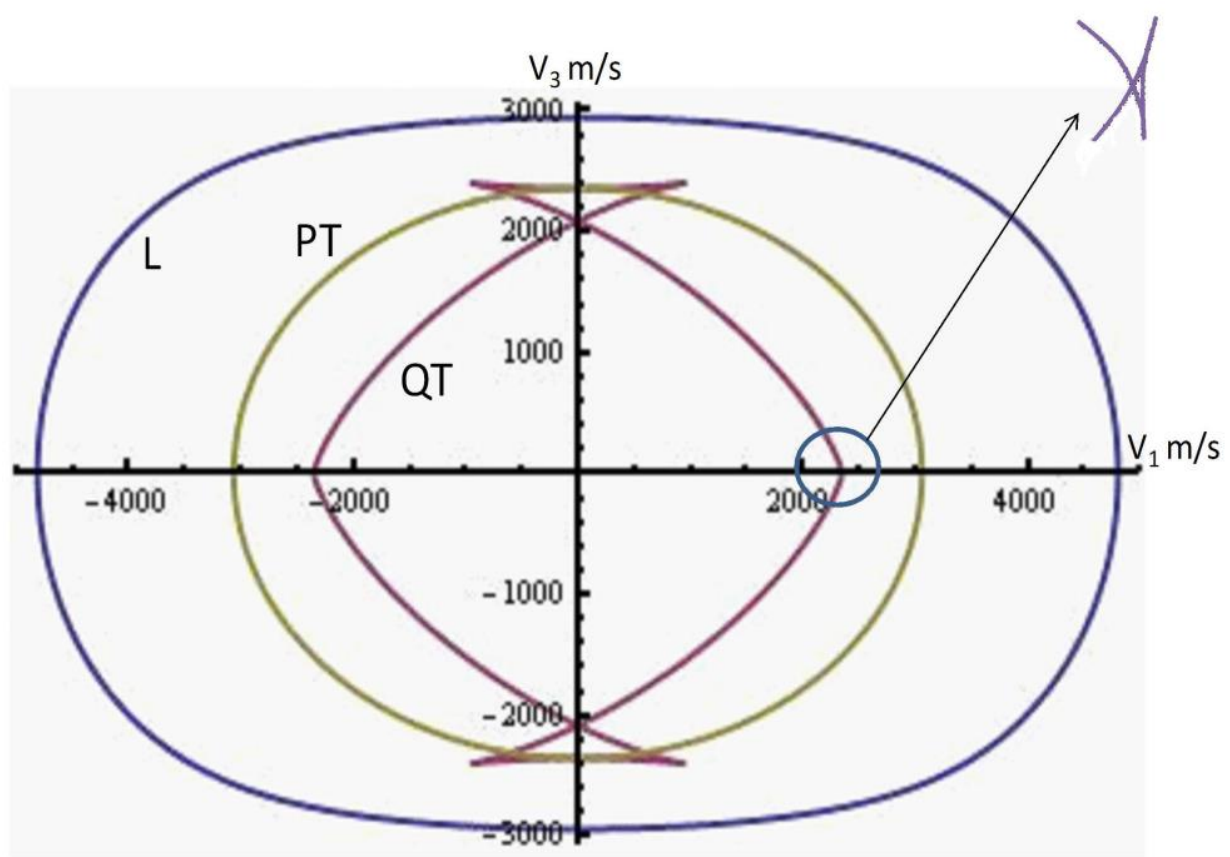

Figure 8. Section of group velocity surface of zinc. 
The prominent features in the angular dependence of $l$ can be understood on the basis of the shape of the outer quasi-transverse sheets of the slowness surface and corresponding folded sheet of the wave surface of zinc depicted in Figures 5a and 8 respectively. We discuss below the singular dependence at the external conical refraction caustic at $\psi=0$, the kink at the fold caustic at $\psi=0.38$ radians $\left(21.81^{\circ}\right)$, and what we interpret as a near singular section of a cusp caustic at $\psi=\pi / 2$ radians $\left(90^{\circ}\right)$.

\subsection{The External Conical Refraction Caustic}

The steep increase of $\ell$ in Fig. 7 towards $\psi=0$ is associated with the external conical refraction caustic. Figure 9 shows the variation of $\ell$ for small $\psi$. Overlaying this curve and virtually indistinguishable from it, is the curve

$\ell=0.535-0.2997 \ln (\psi)$,

$\psi$ in radians, confirming the logarithmic divergence.

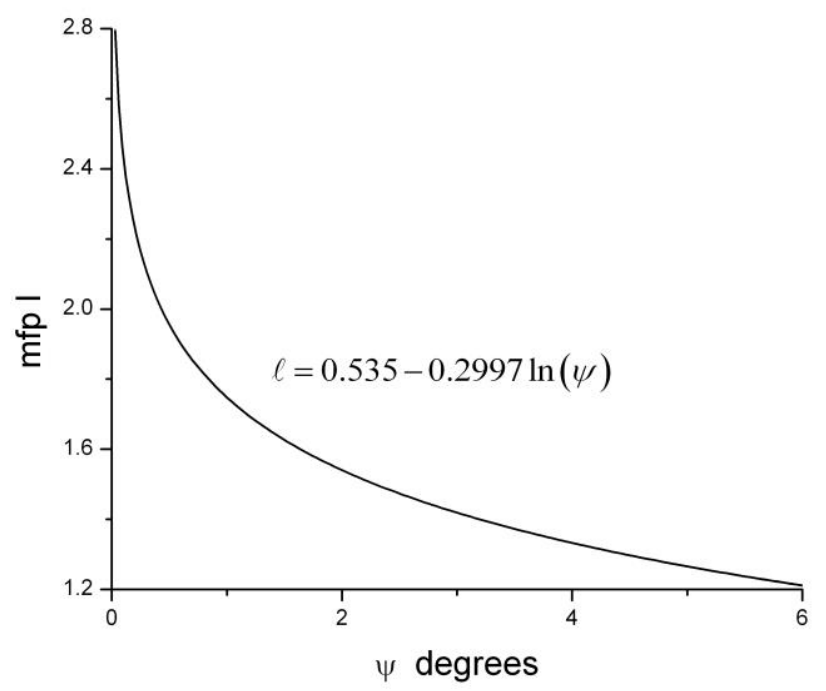

Figure 9. Normalized phonon mean free path $l$ for a zinc rod near the external conical refraction caustic along the zonal axis.

\subsection{The Fold Caustic}

The kink at $\psi_{0}=0.38$ radians $\left(21.81^{\circ}\right)$, shown on an expanded scale in Fig. 10 is a square root singularity associated with the prominent fold and fold caustic in the QT branch at that angle see Figure 1. Inside the folded region the variation of $\ell$ with $\psi$ is linear, and outside there is a square root fall off with angular displacement. Overlaying this curve and virtually indistinguishable from it, is the curve

$\ell=0.86746-0.025 .\left(\psi-\psi_{0}\right)^{1 / 2} \cdot H\left(\psi-\psi_{0}\right)-0.00963 .\left(\psi-\psi_{0}\right)$,

$\psi$ in radians, confirming the nature of the singularity. Note that in this case $\ell$ is not a maximum in the direction of the fold. 


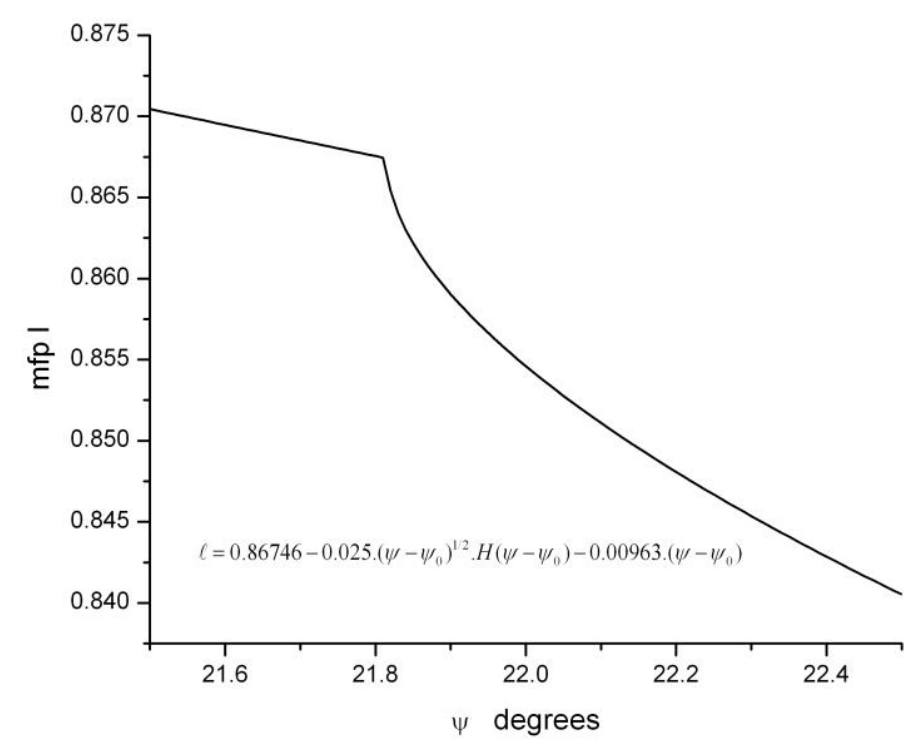

Figure 10. Normalized phonon mean free path $l$ for a zinc rod near the fold caustic.

\subsection{Interpretation of the Behavior of $\ell$ Near $\psi=90^{\circ}$ as the Near Singular Section of a Cusp Caustic}

The steep rise of $\ell$ near $\psi=\pi / 2=90^{\circ}$ is associated with the extensive relatively flat region of the QT sheet of the slowness surface of Zn evident in Fig. 5a, slightly indented to form a waist, where the principal curvature in the $\psi$ direction is small and negative. Figure 11 shows the variation of $\ell$ on an expanded scale, -the flat-topped curve. The wave surface has two folds symmetrically positioned very close on either side of $\psi=\pi / 2$, corresponding to circles on the slowness surface separating the waist from surrounding convex regions. This accounts for the two kinks to the $\ell$ variation bordering the fairly flat region. It requires only a slight change in the elastic constants to bring these two folds into coincidence and then their elimination. For the elastic constants to satisfy the relationship

$$
\frac{\left(C_{13}+C_{44}\right)^{2}}{C_{33}\left(C_{11}-C_{44}\right)}=1 \text {, }
$$

which requires reducing $C_{44}$ from $39.6 \mathrm{GPa}$ to $38.44 \mathrm{GPa}$ (a $2.9 \%$ reduction), this renders the curvature at $\psi=\pi / 2$ zero, and transforms the flat-topped curve into the cusped curve. Overlaying this curve and almost indistinguishable from it is the curve

$$
\ell=1.864-1.006|\psi-\pi / 2|^{1 / 3} \text {. }
$$

This is readily interpreted in the context of the catastrophe theory classification of caustics [37] as the behavior of the cusp caustic, in which the control parameter in the direction of the cusp is $C_{44}$, and the control parameter perpendicular to that direction is the angle $\psi$. The azimuthal angle $\phi$ is an ignorable coordinate in this context. A further $2.9 \%$ reduction in $C_{44}$ to $37.28 \mathrm{GPa}$ yields a non-singular section lying beyond the cusp point, the smooth curve in Fig. 11 . 


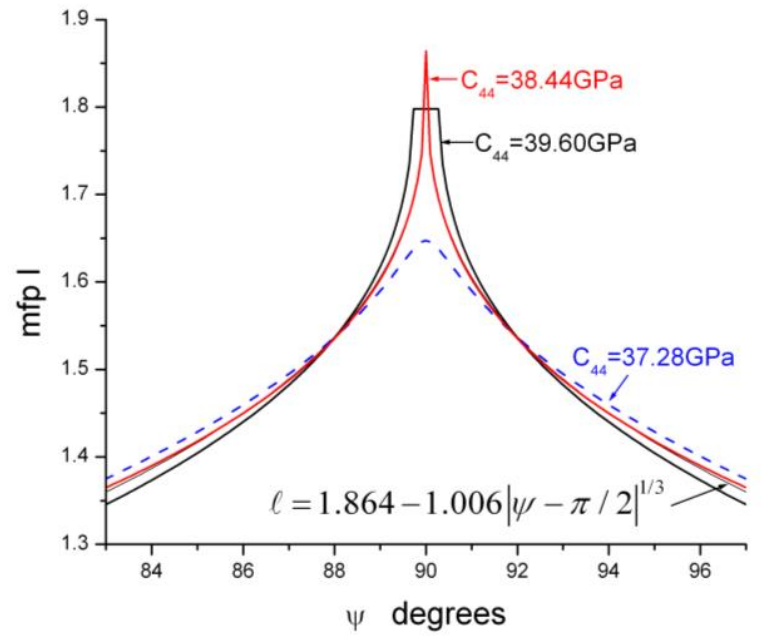

(a)

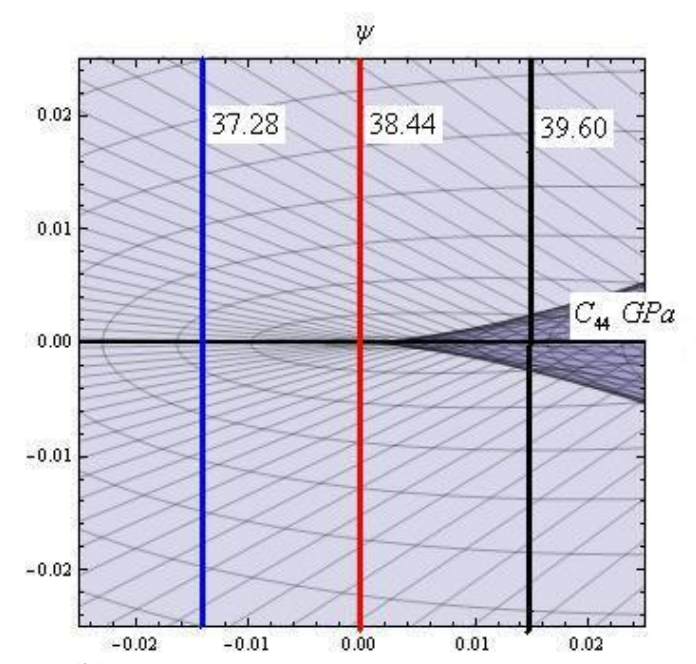

(b)

Figure 11. (a) Normalized phonon mean free path $l$ for a zinc rod oriented near $\psi=90^{\circ}$, (b) schematic of cusp sections, with vertical direction representing angular deviation, and horizontal direction value of $C_{44}$.

A similar situation prevails for cubic crystals such as $\mathrm{Si}$ and Ge, for which the FT sheet of the slowness surface is folded across the $\{100\}$ planes. This is accompanied by a pair of line caustics symmetrically disposed on either side of the $\{100\}$ planes, and which run between adjacent $<100>$ directions. [26]. With decreasing $C_{44}$, as the elastic constants approach the condition [20]

$$
\frac{2\left(C_{12}+C_{44}\right)^{2}}{C_{11}\left(C_{11}+C_{12}\right)}=1 \text {, }
$$

these caustics move closer together, and then coalesce and disappear (for $\mathrm{Si}$ this requires a $6.96 \%$ reduction of $C_{44}$ from 79.56 to $74.02 \mathrm{GPa}$ for this to happen). For a crystal satisfying the condition (59), the angular dependence of $\ell$ with deviation of the rod axis from the [110] direction in the $(1 \overline{1} 0)$ plane exhibits $1 / 3$ power law singular behavior.

\section{Conclusions}

We have extended the Casimir model for boundary-limited thermal conductivity to the case of crystalline rods with axes aligned close to phonon-focusing caustics. The conductivity has been shown to diverge logarithmically on approach to an external conical refraction caustic, a caustic displayed by hexagonal crystals conforming to certain conditions on their elastic constants. The Casimir limit has been shown not to exist in the direction of the caustic, meaning that in the absence of bulk scattering the conductivity of an infinitely long rod would be infinite. For fold and cusp caustics, which are generic to most crystals, the conductivity has been shown to remain finite in the caustic direction. Inside the fold caustic, the conductivity varies linearly with angle $|\psi|$ away from the caustic, whereas outside the caustic it exhibits in addition a singular falloff proportional to $-|\psi|^{1 / 2}$. The Casimir conduction is not necessarily a maximum in the direction of 
a fold caustic, as seen in the example of zinc. Within the cusp caustic, the conductivity also varies linearly with angle $|\psi|$ away from the caustic, whereas on the outside of the caustic it exhibits in addition a singular component proportional to $-|\psi|^{1 / 3}$ normal to the cusp direction, and proportional to $-|\psi|^{1 / 2}$ in the direction of the cusp. Calculations for a zinc rod reveal the expected behavior of the effective mean free path near the external conical refraction and fold caustics, and the behavior near the equatorial plane can be interpreted as the near-singular section of a cusp caustic.

Many other physical processes influence the thermal conductivity of rods and nanowires at low temperatures, and these need to be taken into account in interpreting measurements. These include the effect of finite rod length, the shape of the cross section, the partial angular- and frequency-dependent specularity at the surface, remnant bulk scattering and phonon dispersion. This last item, in particular, is important for the case of nanowires, for which boundary scattering can be important even above the Debye temperature. While recognizing the importance of these factors, we have focused on a matter of principle regarding the role of phonon focusing caustics in the Casimir conduction.

It is hoped that the clearer understanding of the role of phonon focusing in boundary-limited thermal conduction will aid in the interpretation of numerical simulations and experimental results, and also in the choice of materials and orientations for investigation and potential applications.

\section{Acknowledgments}

The authors are greatly indebted to O. B. Wright for his critical reading of the manuscript. AGE thanks the South African National Research Foundation for financial support. The contribution by AAM was supported as part of the S3TEC Energy Frontier Research Center funded by the U.S. Department of Energy, Office of Science, Office of Basic Energy Sciences under Award No. DE-SC0001299.

\section{References}

[1] H. B. G. Casimir, Physica 5, 495 (1938).

[2] P. G. Klemens, in Solid State Physics Eds. F. Seitz and D. Turnbull (Academic Press, New York, 1958), Vol. 7, pps.1-98.

[3] W. J. deHaas and T. Biermasz, Physica 5, 47 (1938).

[4] J. W. Vandersande, Phys. Rev. B 13, 4560 (1976).

[5] D. G. Cahill, W. K. Ford, K. E. Goodson, G. D. Mahan, H. J. Maris, A. Majumdar, R. Merlin, and S. R. Phillpot, J. Appl. Phys. 93, 793 (2003).

[6] D. G. Cahill, P. V. Braun, G. Chen, D. R. Clarke, S. Fan, K. E. Goodson, P. Keblinski, W. P. King, G. D. Mahan, A. Majumdar, H. J. Maris, Simon R. Phillpot, E. Pop, and L. Shi, Appl. 
Phys. Rev. 1, 011305 (2014).

[7] A. M. Marconnet, M. A. Panzer and K. E. Goodson, Rev. Mod. Phys. 85, 1295 (2013).

[8] R. Chen, A. I. Hochbaum, P. Murphy, J. Moore, P. Yang, and A. Majumdar, Phys. Rev. Lett. 101, 105501 (2008).

[9] J. P. Feser, J. S. Sadhu, B. P. Azeredo, K. H. Hsu, J. Ma, J. Kim, M. Seong, N. X. Fang, X. Li, P. C. Ferreira, S. Sinha and D. G. Cahill, J. Appl. Phys. 112, 114306 (2012).

[10] F. Zhou, A. L. Moore, J. Bolinsson, A. Persson, L. Froberg, M. T. Pettes, H. Kong, L. Rabenberg, P. Caroff, D. A. Stewart, N. Mingo, K. A. Dick, L. Samuelson, H. Linke and L. Shi, Phys. Rev. B 83, 205416 (2011).

[11] H. J. Maris and S. I. Tamura, Phys. Rev. B 85, 054304 (2012).

[12] W. Li and N. Mingo, J. Appl. Phys. 114, 054307 (2013).

[13] Z. Aksamija and I. Knezevic, Phys. Rev. B 82, 045319 (2010).

[14] J. S. Heron , T. Fournier, N. Mingo, and O. Bourgeois, Nano Lett. 9, 1861 (2009).

[15] C. Blanc, A. Rajabpour, S. Volz, T. Fournier, and O. Bourgeois, Appl. Phys. Lett. 103, 043109 (2013).

[16] J.B. Hertzberg, M. Aksit, O.O. Otelaja, D.A. Stewart, R.D. Robinson, Nano Lett. 14, 403 (2014).

[17] A. K. McCurdy, H. J. Maris and C. Elbaum, Phys. Rev. B 2, 4077 (1970). Note that in this paper the symbol $s$ is used for phase velocity, not slowness.

[18] H. J. Maris, J. Acoust. Soc. Am. 50, 812 (1971).

[19] H. J. Maris, in Nonequilibrium Phonons in Nonmetallic Crystals, W. Eisenmenger and A.A. Kaplyanskii, Eds. (North Holland, Amsterdam, 1986), pps. 51-90.

[20] A. G. Every, Phys. Rev. B24, 3456 (1981).

[21] J. P. Wolfe, Imaging Phonons (Cambridge University Press, Cambridge, 1998).

[22] A. G. Every, A. A. Maznev, W. Grill, M. Pluta, J. D. Comins, O. B. Wright, O. Matsuda, W Sachse and J. P. Wolfe, Wave Motion 50, 1197 (2013).

[23] C. G. Winternheimer and A. K. McCurdy, Solid State Commun. 14, 919 (1974).

[24] A. K. McCurdy, Phys. Rev. B 9, 466 (1974).

[25] C. G. Winternheimer and A. K. McCurdy, Phys. Rev. B 18, 6576 (1978).

[26] A. K. McCurdy, Phys. Rev. B 26, 6971 (1982).

[27] I. I. Kuleyev, I. G. Kuleyev, S. M. Bakharev and A. V. Inyushkin, Physica B 416, 81 (2013).

[28] I. I. Kuleyev, I. G. Kuleyev, S. M. Bakharev and A. V. Inyushkin, Phys. Solid State 55, 
1545 (2013).

[29] I. I. Kuleyev, I. G. Kuleyev, S. M. Bakharev and A. V. Inyushkin, Phys. Solid State 55, 31 (2013).

[30] M. B. Koss, K. A. McCarthy and H. H. Sample, Phys. Rev. B 42, 5822 (1990).

[31] T. Markussen, A.-P. Jauho and M. Brandbyge, Nano Letters 8, 3771 (2008).

[32] A. V. Inyushkin, A. N. Taldenkov, A. V. Gusev, A. M. Gibin, V. A. Gavva and E. A. Kozyrev, Phys. Solid State 55, 235 (2013).

[33] A. G. Every and K. Y. Kim, J. Acoust. Soc. Am. 95, 2505 (1994).

[34] M. J. P. Musgrave, Crystal Acoustics (Acoustical Society of America, 2003).

[35] V. T. Buchwald, Proc. R. Soc. London Ser. A 253, 563 (1959).

[36] H. J. Maris, Phys. Rev. B 28, 7033 (1983).

[37] M. V. Berry, Adv. in Phys. 25, 1 (1976).

[38] A. G. Every, Phys. Rev. B34, 2852 (1986).

[39] K. Y. Kim, W. Sachse and A. G. Every, Phys. Rev. Lett. 70, 3443 (1993).

[40] K. Y. Kim, W. Sachse and A. G. Every, Int. J. Mod. Phys B 8, 2327 (1994).

[41] A. G. Every, , in Handbook of Elastic Properties of Solids, Liquids, and Gases, Ed. Levy M., Bass H., Stern R. (New York: Academic Press; 2001), Vol 1, Chap.1, pp. 3 - 36.

[42] A. G. Every and A. K. McCurdy, Second and Higher Order Elastic Constants, LandoltBörnstein, New Series Group III, Vol 29a, Berlin; Springer (1992). 